\title{
Charged Higgs Prospects with ATLAS
}

\author{
Martin Flechl* on behalf of the ATLAS Collaboration \\ Uppsala Universitet \\ E-mail: martin.flechl@cern.ch
}

The charged Higgs boson simulation studies for charged Higgs boson searches with ATLAS are presented. Since the previous workshop of this series in 2006, the first studies with a realistic detector simulation for the most important charged Higgs boson channels have been carried out, including all trigger levels and taking the dominant systematic uncertainties into account. These studies deal both with low-mass (produced in top quark decays) and high-mass $\mathrm{H}^{+}$(produced in $g g$ - and $g b$-fusion), with a consistent treatment of the transition region. Analysis techniques and their largest challenges are discussed. The ATLAS $H^{+}$sensitivity is presented in terms of discovery and exclusion contours, and a brief outlook on the LHC data-taking era is given.

Prospects for Charged Higgs Discovery at Colliders

16-19 September 2008

Uppsala, Sweden

* Speaker. 


\section{Introduction}

Charged Higgs bosons are naturally predicted in many non-minimal Higgs scenarios, such as Two Higgs Doublet Models (2HDMs), and models with Higgs triplets including Little Higgs models. Their discovery would be a definite signal for the existence of New Physics beyond the Standard Model (SM), possibly the first experimental evidence to be found if the Minimal Supersymmetric Standard Model (MSSM) is realized in nature, and the supersymmetry mass scale is sufficiently high such that sparticles escape discovery.

The results shown here focus on the 2HDM, in particular in the context of the MSSM which employs a so-called type II-2HDM. The MSSM Higgs sector contains five Higgs bosons: one light and one heavy CP-even scalar $\left(h^{0}, H^{0}\right)$, one CP-odd scalar $\left(A^{0}\right)$, and finally two charged scalars $\left(H^{ \pm}\right)^{1}$. None of the masses is a priori predicted by the theory, but at tree level the Higgs sector of the MSSM can be completely determined by two parameters in addition to those of the SM. These are usually taken to be the mass of a Higgs boson, e.g. the charged Higgs boson mass $m_{H^{+}}$, and the ratio of the two Higgs doublet vacuum expectation values $(\tan \beta)$. Owing to the large number of parameters, MSSM models are usually studied in benchmark scenarios where the Supersymmetry (SUSY) parameters are chosen to obtain specific phenomenologies. One such benchmark scenario is the $m_{h}$-max scenario where the mass of the lightest neutral Higgs boson is maximized. This $m_{h}$-max scenario is implied in the figures and results shown in the following, unless mentioned otherwise.

The search strategies for charged Higgs bosons depend on its hypothesized mass, which dictates both the production and the available decay modes. Below the top quark mass the main production mode is through top quark decays, $t \rightarrow H^{+} b$, and in this range the $H^{+} \rightarrow \tau \nu$ decay mode is dominant for $\tan \beta>3$. Above the top quark threshold, production mainly takes place through $\mathrm{gb}$ fusion $\left(\mathrm{g} b \rightarrow t \mathrm{H}^{+}\right)$, and for such high charged Higgs boson masses the decay into a top and $\mathrm{a} \mathrm{b}$ quark dominates, $H^{+} \rightarrow t \bar{b}$. At present, an absolute lower bound on the charged Higgs boson mass of about $80 \mathrm{GeV}$ exists from LEP [1], independently of the charged Higgs boson branching ratios (BR). The Tevatron collider at Fermilab has excluded the charged Higgs boson near the LEP mass limit of about $80 \mathrm{GeV}$ for small $(\lesssim 1.5)$ and large $\tan \beta$ values ( $\gtrsim 30)$ in the MSSM [2].

The LHC (Large Hadron Collider) at CERN is a proton-proton collider with a design centerof-mass energy of $14 \mathrm{TeV}$. It will give access to a large portion of the 2HDM and MSSM parameter space. The ATLAS detector is one of the two general-purpose experiments at the LHC. It has full solid-angle coverage and will serve to analyze proton-proton collisions starting in 2009. Its major components are the Inner Detector (the tracking system consisting of three subdetectors), the Liquid Argon Electromagnetic Calorimeter, the TileCal Hadronic Calorimeter and the Muon Spectrometer.

This note presents the preparations of the ATLAS experiment for the $\mathrm{H}^{+}$search and is organized as follows: Section 2 outlines the trigger and reconstruction tools as well as the simulation details, Sections 3 and 4 describe the searches for charged Higgs bosons with masses below and above the top quark mass, respectively. Systematic uncertainties are discussed and combined results are shown in Section 5.

\footnotetext{
${ }^{1}$ In the following, the charged Higgs boson will be denoted $H^{+}$, but $H^{-}$and charge conjugate processes are always implicitly included.
} 


\section{Trigger, Reconstruction and Simulation Details}

In accordance with the ATLAS trigger [3] menu for low luminosity $\left(L=2 \cdot 10^{33} \mathrm{~cm}^{-2} \mathrm{~s}^{-1}\right)$ runs, the following $H^{+}$trigger menu [4] has been identified:

- high- $p_{T}$ lepton (electron or muon)

- lepton plus missing transverse energy

- high missing tranverse energy

- $\tau$ jet [5] plus missing transverse energy (optionally, plus additional jets)

This menu has been chosen considering the characteristics of $\mathrm{H}^{+}$events and meets the requirements of the trigger bandwidth budget. Charged Higgs boson studies also depend on a number of highlevel reconstruction tools. Considering the $\mathrm{H}^{+}$decay modes, the most important ones are $\tau$-tagging and $b$-tagging and are discussed in detail in these proceedings $[6,7]$.

The studies [8] presented in the following sections have been performed using a realistic simulation of the ATLAS Detector. Large samples of signal events have been produced for several mass points as well as different production and decay modes. Depending on the signature, a subset of the following backgrounds has been considered: Standard Model-like top quark pair production $(t \bar{t}), W+$ jets, single top quark production, and QCD dijet events. Details of the event generation and simulation steps can be found in Reference [8]. All cross section calculations used for signal and background are NLO and for the case of the $\mathrm{H}^{+}$events the dominating SUSY corrections [9] are taken into account. All MSSM plots shown here are based on the $m_{h}$-max benchmark:

$$
m_{h} \text {-max: } M_{S U S Y}=500, A_{t}=1000, \mu=200, M_{2}=1000, M_{3}=1000(\text { all units are GeV) }
$$

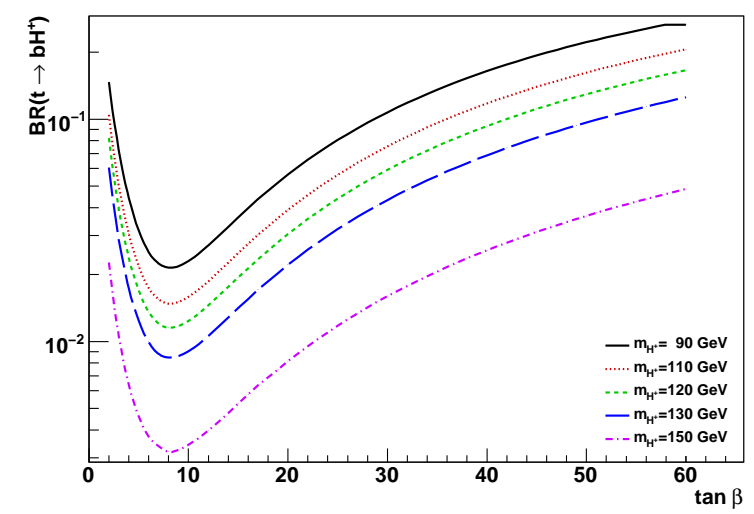

Figure 1: The Branching Ratio $t \rightarrow b H^{+}$as a function of $\tan \beta$ for several $H^{+}$mass points in the $m_{h}$-max scenario [10]. 


\section{Charged Higgs Boson Searches In Top Quark Pair Events}

The main production mode for $H^{+}$at the LHC for $m_{H^{+}}<m_{t}$ is the decay of a top quark $t \rightarrow b H^{+}$ in $t \bar{t}$ events since the $t \bar{t}$ cross section is large (833 pb at NLO [11]). The production of about 8 million top quark pairs per year at the LHC at low luminosity $\left(\mathscr{L}=10 \mathrm{fb}^{-1}\right.$ per year) is anticipated and with a typical expected branching ratio $t \rightarrow b H^{+}$of a few percent this implies a sizeable $H^{+}$ production. The branching ratio $t \rightarrow b H^{+}$as a function of $\tan \beta$ for different values of $m_{H^{+}}$is shown in Figure 1.

Recent searches have focused on hadronic $\tau$ decays $\left(\tau_{H}\right)$ of the $H^{+}$while both hadronic and semileptonic decays of the second top quark in the event are considered, and on leptonic $\tau$ decays $\left(\tau_{L}\right)$ with hadronic decays of the second top quark.

3.1 $H^{+} \rightarrow \tau_{H} v, W \rightarrow q q$

This channel has previously been investigated for ATLAS with a parametrized detector simulation [12] and has now for the first time been investigated with a realistic detector simulation [13]. Its event signature consists of a $\tau$ jet, missing transverse energy, $2 b$ jets and a hadronic $\mathrm{W}$ and top quark which can be reconstructed. Since all the neutrinos in the hard event are associated to the $\mathrm{H}^{+}$decay the transverse component of this boson can be reconstructed. The main challenge is the trigger as there are no leptons in the event. The backgrounds considered are $t \bar{t}$, QCD dijets, $W+$ jets and single top.
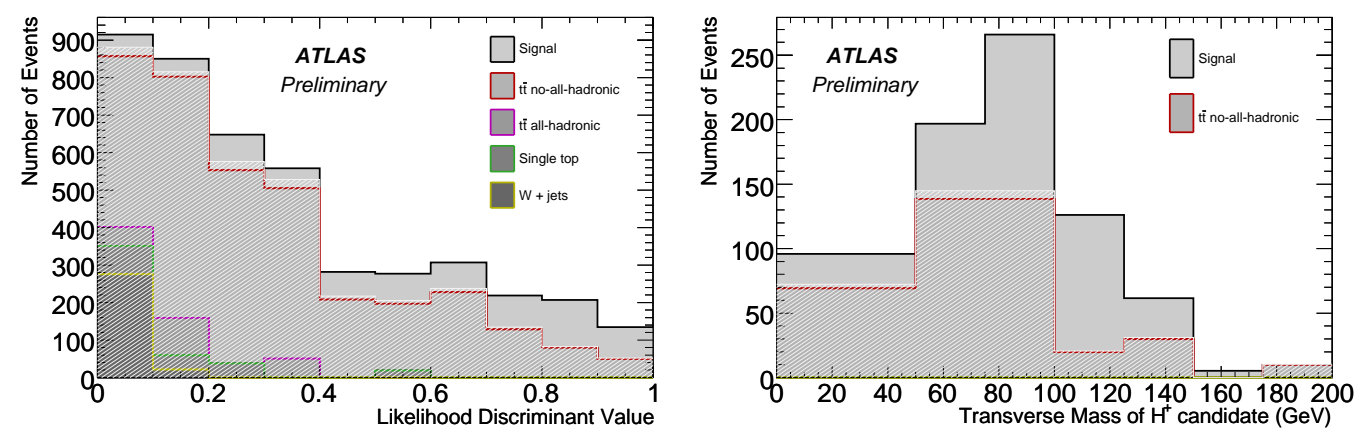

Figure 2: Left: Likelihood discriminant value. Right: Transverse mass of the $H^{+}$candidate. Both figures show the signal $\left(m_{H^{+}}=130 \mathrm{GeV}, \tan \beta=20\right)$ and the corresponding dominant backgrounds.

The $\tau$ jet plus missing $E_{T}$ trigger signature is used, leading to an efficiency for the signal of only about $10 \%$ (the exact value depends on the $H^{+}$mass). This is followed by offline preselection cuts requiring exactly $1 \tau$ jet and $2 b$ jets, at least 2 light jets, substantial missing $E_{T}$, and no isolated lepton in the event. Furthermore, the reconstruction of the hadronic $\mathrm{W}$ and top quark within mass windows is required. The top quark on the $H^{+}$side is reconstructed in the transverse plane from the other $b$ jet, the $\tau$ jet and the missing transverse momentum. Selection cuts are then applied to relations between the two top quarks: their $p_{T}$ ratio has to be smaller than 2 , and the angle between their transverse momenta larger than $2.5 \mathrm{rad}$. These cuts enhance the $t \bar{t}$ topology (signal and background!) but effectively reduce all other backgrounds. 
In the next step a likelihood is built with the aim to suppress $t \bar{t}$ events. The probability density functions used for the likelihood are transformations of the following variables: $p_{T}$ ratios: (1) hardest $\tau$ track and $\tau$ jet, (2) $\tau$ jet and $b$ jet; angles: (3) $\tau$ jet and missing $E_{T}$, (4) $H^{+}$and $b$ jet, (5) $\tau$ jet and $b$ jet; mass: (6) $\tau$ jet- $b$ jet pair. Additionally, (7) the product of variables 5 and 6 is used. For all variables, the $b$ jet in question is the one from the $\mathrm{H}^{+}$side of the event.

The resulting likelihood discriminant value for signal and backgrounds is shown in Figure 2, left. Events with a likelihood value greater than 0.6 are kept (for $m_{H^{+}}>140 \mathrm{GeV}$, the requirement is increased to 0.8). The transverse mass of the $\mathrm{H}^{+}$candidate is calculated from the remaining events (see Figure 2, right). The shape of this distribution is used to extract the significance of the signal, using the profile likelihood method [14]. The resulting discovery and exclusion potential is shown in Figure 3. The discovery potential covers a sizeable portion of the MSSM parameter space but has a hole at intermediate $\tan \beta \approx 3-20$ where the production cross section is low. However, the exclusion sensitivity extends into this region.
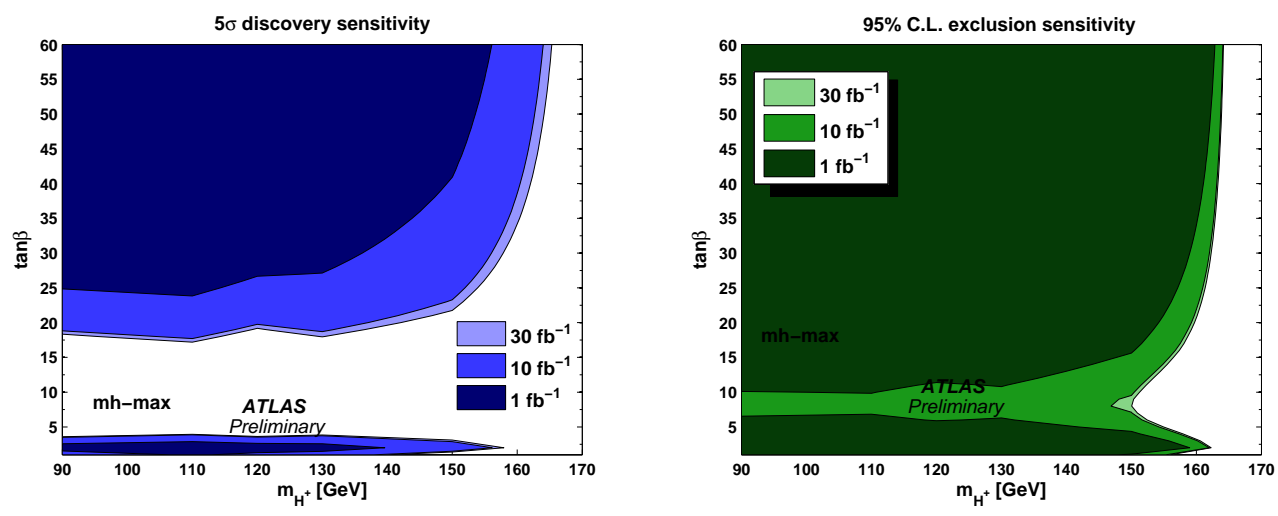

Figure 3: ATLAS $H^{+}$discovery and exclusion sensitivity for searches in the $t \bar{t} \rightarrow b \tau_{H} v b q q$ mode for the $m_{h}$-max scenario. Dominant systematic and statistical uncertainties are included (see Section 5).

3.2 $\mathrm{H}^{+} \rightarrow \tau_{L} v, W \rightarrow q q$

This channel is investigated in ATLAS for the first time. The signal signature consists of a lepton, missing energy, $2 b$ jets and a hadronic $W$ and top quark which can be reconstructed. The backgrounds considered are $t \bar{t}, W+\mathrm{jets}$ and QCD dijets. The advantage of this channel is that the lepton facilitates triggering on its events. Since all neutrinos have the same source $\left(\mathrm{H}^{+}\right)$, the transverse charged Higgs mass can actually be extracted by generalizing the transverse mass concept [15].

Events considered are those triggered by a lepton plus missing $E_{T}$ trigger or a high missing $E_{T}$ signature. In the preselection, a lepton is required, at least four jets of which at least two are required to be $b$-tagged, and missing $E_{T}>120 \mathrm{GeV}$. After preselection, all backgrounds except for $t \bar{t}$ are negligible.

In the next step, variables to discriminate the signal against $t \bar{t}$ are considered. The hadronic top quark is reconstructed by first finding the dijet closest to the $W$ mass. Then the two $b$ jets are assigned to the $W$ and the $H^{+}$by means of a likelihood using three variables ( $b$ jet charge, angle $b$ jet-lepton, angle $b$ jet- $W$ ). The mass of the reconstructed hadronic top quark is then required to be 
within a mass window of $100-300 \mathrm{GeV}$. Then the quantity $\cos \psi=2 m_{\ell b}^{2} /\left(m_{t}^{2}-m_{W}^{2}\right)$ is calculated and required to be smaller than - 0.8 . In events where the lepton is directly produced in the $W$ decay, $\psi$ is the angle between the lepton and top quark (in the $W$ rest frame, in the limit of a massless $b$ ). The cut thus helps to suppress these events, together with the following requirement on m(lepton, $E_{T}^{\text {miss }}$ ) to be lower than $60 \mathrm{GeV}$.
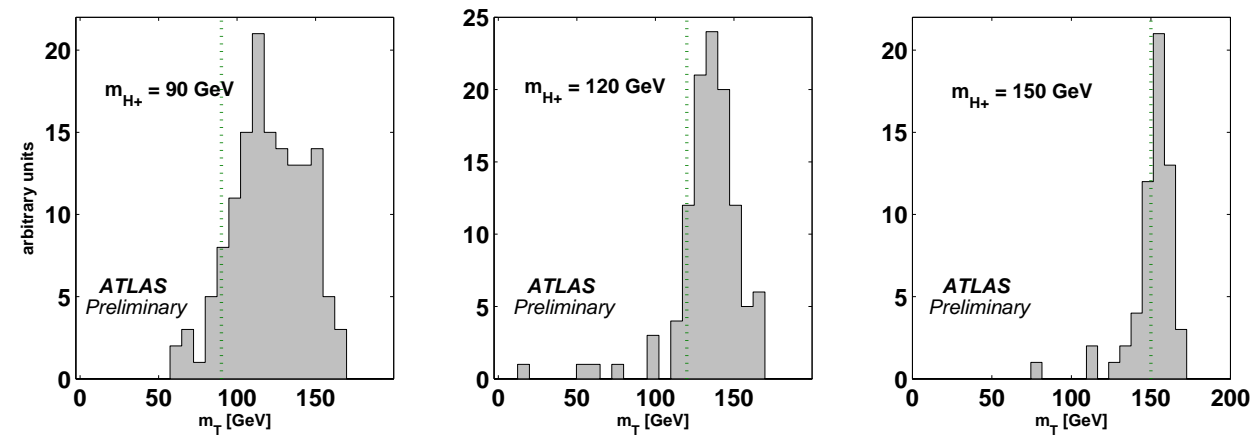

Figure 4: Reconstructed transverse $H^{+}$mass for the signal, for three different nominal $H^{+}$mass cases.

From the remaining events, the generalized transverse mass is reconstructed using a novel technique [15] developed in the course of preparing this analysis:

$$
\left(m_{T}^{H^{+}}\right)^{2}=\left(\sqrt{m_{t o p}^{2}+\left(\vec{p}_{T}^{l e p}+\vec{p}_{T}^{b}+\vec{p}_{T}^{m i s s}\right)^{2}}-p_{T}^{b}\right)^{2}-\left(\vec{p}_{T}^{m i s s}+\vec{p}_{T}^{l e p}\right)^{2}
$$

The resulting transverse charged Higgs boson mass distributions for several nominal $\mathrm{H}^{+}$values are shown in Figure 4 and show a good agreement. The resulting significance is then calculated either from the reconstructed $\mathrm{H}^{+}$or $W$ mass, depending on which performs better for the concerned $\mathrm{H}^{+}$ mass range. Examples of these two distributions are shown in Figure 5.
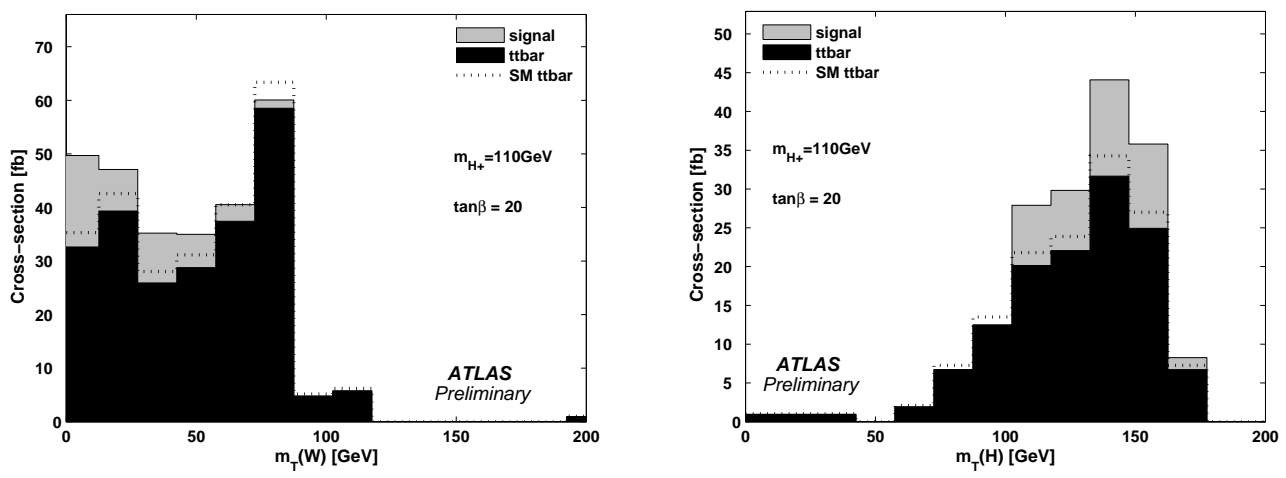

Figure 5: Reconstructed transverse $W$ (left) and $H^{+}$(right) mass for the example of a charged Higgs boson mass of $110 \mathrm{GeV}$ and $\tan \beta=20$. The signal and background case are shown, stacked on each other and normalized to their expected cross section.

The resulting discovery and exclusion potential is shown in Figure 6. The sensitivity is less than in the channel with hadronic $\tau$ decays, however, it contributes substantially to the combined 
$H^{+}$sensitivity and is more robust since the lepton reconstruction is more straight-forward than the reconstruction of a $\tau$ jet, both for the trigger and the offline reconstruction.
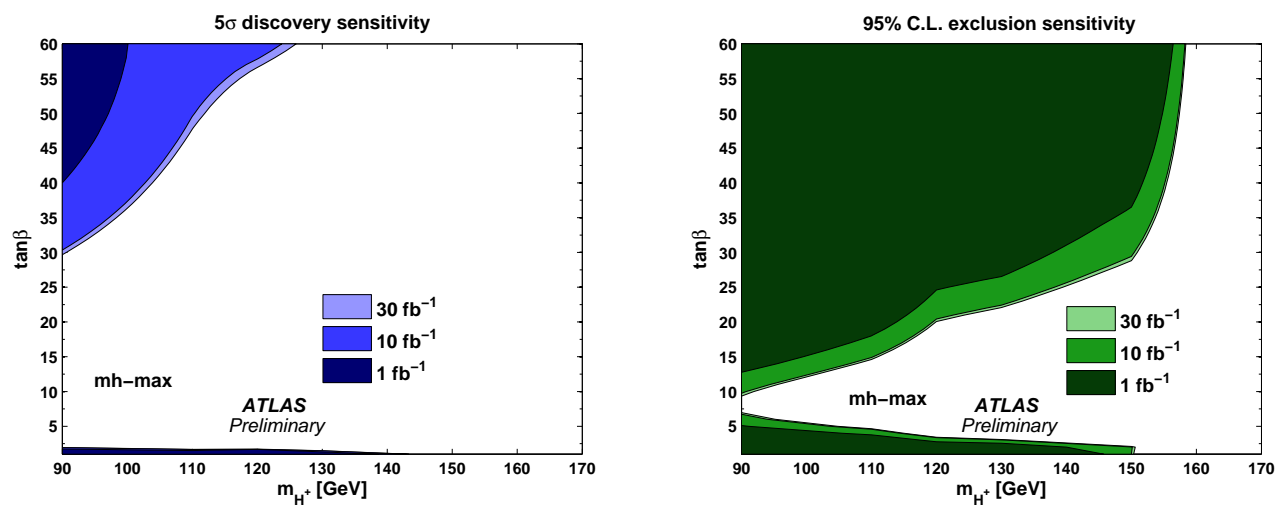

Figure 6: ATLAS $H^{+}$discovery and exclusion sensitivity for searches in the $t \bar{t} \rightarrow b \tau_{L} v b q q$ mode for the $m_{h}$-max scenario. Dominant systematic and statistical uncertainties are included (see Section 5).

\section{3 $H^{+} \rightarrow \tau_{H} v, W \rightarrow \ell v$}

This channel has not been studied for ATLAS for many years and has only recently been reinvestigated[16]. The event signature consists of a lepton, missing $E_{T}$, a $\tau$ jet and two $b$ jets. Due to the presence of neutrinos from multiple sources the reconstruction of the $H^{+}$is not possible. This is also the case for the $W$ and top quarks in the event. The backgrounds considered are $t \bar{t}$, single top and $W+$ jets.
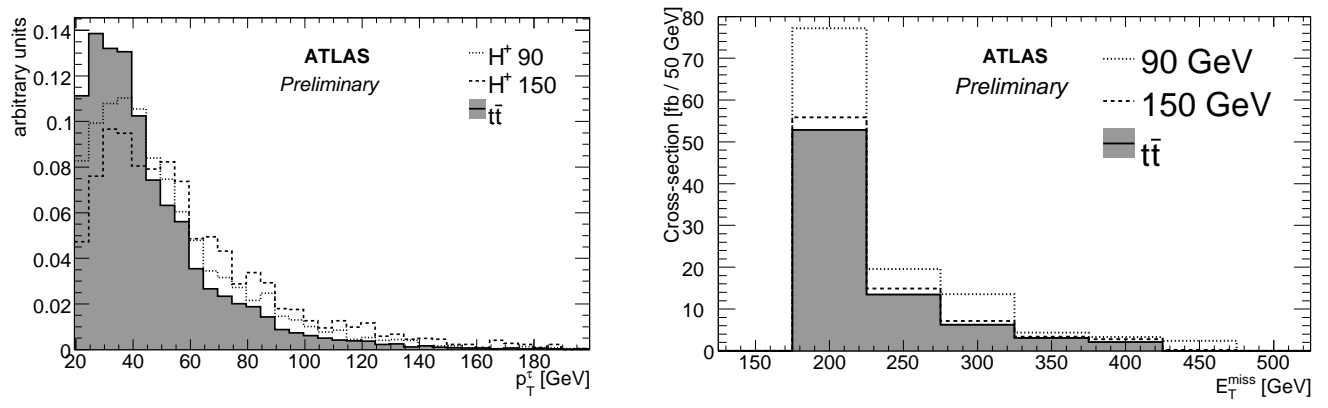

Figure 7: Left: Distribution of the $\tau$ jet transverse momentum for two signal mass points and the $t \bar{t}$ background. Right: The missing $E_{T}$ distribution for two signal mass points individually stacked on top of the contribution from the $t \bar{t}$ background, normalized to their cross sections for $\tan \beta=20$.

Events are triggered by a combined signature requiring missing $E_{T}$ and either a lepton or a $\tau$ jet. The following selection cuts are applied, following closely the final state of the event: at least one isolated lepton is required, and at least 3 jets. Of these jets, at least one is required to be $b$-tagged and one to be $\tau$-tagged. The transverse momentum of the $\tau$ jet has to be higher than 40 $\mathrm{GeV}$ which helps to discriminate against $t \bar{t}$ events, as can be seen in Figure 7, left. Only events where the charge of the $\tau$ jet is opposite to the lepton charge are accepted. A final cut is placed on 
the missing $E_{T}$, requiring at least $175 \mathrm{GeV}$. The missing $E_{T}$ distribution (see Figure 7, right) is then used to extract the significance.
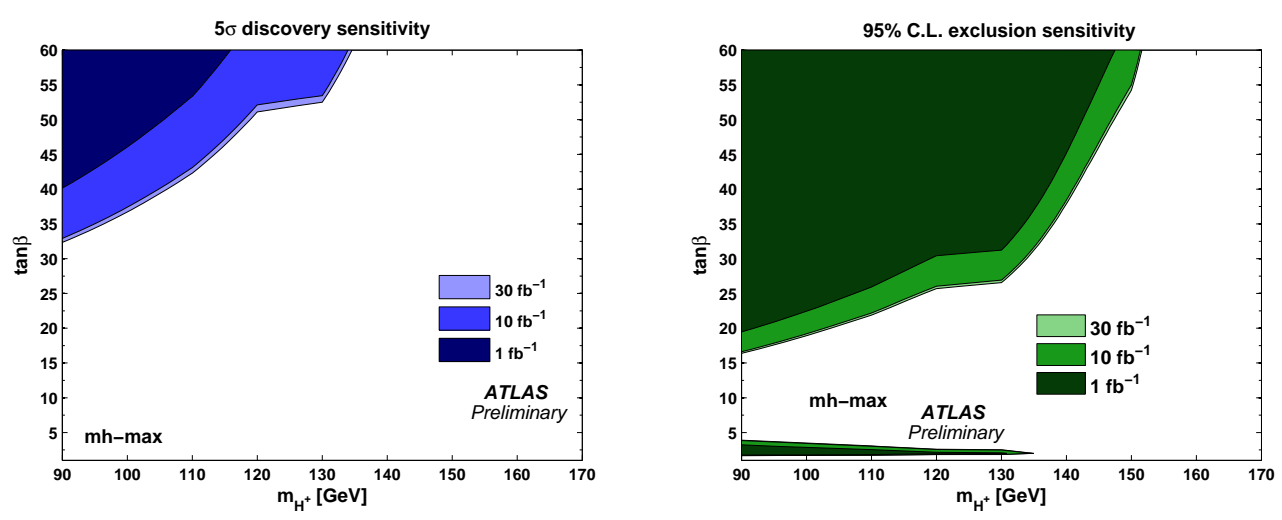

Figure 8: ATLAS $H^{+}$discovery and exclusion Sensitivity for searches in the $t \bar{t} \rightarrow b \tau_{H} v b \ell v$ mode for the $m_{h}$-max scenario. Dominant systematic and statistical uncertainties are included (see Section 5).

The resulting discovery and exclusion contours are shown in Figure 8. Discovery sensitivity is given for low $m_{H^{+}}$and high $\tan \beta$, while exclusion is possible for a large part of the parameter space above $\tan \beta=20$. Although the sensitivity is smaller than for the channel presented in Section 3.1, this constitutes an important contribution to the combined $\mathrm{H}^{+}$sensitivity and an important crosscheck even if the $\mathrm{H}^{+}$is discovered in another channel first.

\section{Charged Higgs Boson Searches in gg- and gb-fusion}

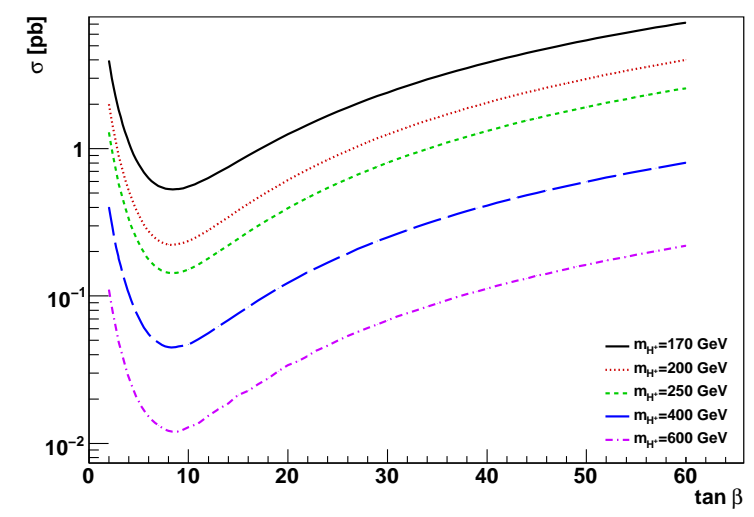

Figure 9: $H^{+}$production cross section in $g g$ - $/ g b$-fusion for the $m_{h}$-max scenario as a function of $\tan \beta$ for various charged Higgs boson mass values [10, 17].

If the charged Higgs boson is heavier than the top quark then it is dominantly produced in $g g$ and $g b$ fusion, $g g \rightarrow t b H^{+}$and $g b \rightarrow t H^{+}$. The typical cross section is of the order of $1 \mathrm{pb}$ or lower, as illustrated in Figure 9. This is much lower than the expected cross sections for a light $\mathrm{H}^{+}$, 
however, the kinematic difference between signal and background increases at the same time and can be exploited.

Above the top quark mass, charged Higgs bosons decay dominantly to $t b$ but the decay to $\tau v$ is still sizeable (the branching ratio is of the order of $10 \%$, depending on $m_{H^{+}}$and $\tan \beta$ ) and offers a much cleaner signature than the $t b$ decay. Charged Higgs boson searches currently focus on these two decay modes.

\section{1 $H^{+} \rightarrow \tau v$}

This mode has previously been investigated for ATLAS with a realistic detector simulation for the signal and a parametrized detector simulation for the background [18]. The event signature consists of a hard $\tau$ jet, large missing $E_{T}, 1-2 b$ jets and a hadronic $W$ and top quark which can be reconstructed. Such a heavy $\mathrm{H}^{+}$can be separated from the background quite well, however, its production cross section is small and decreases quickly with $m_{H^{+}}$. The backgrounds considered are $t \bar{t}$, single top, $W+$ jets and QCD dijet.

The $\tau$ jet plus missing $E_{T}$ signature is used, leading to an efficiency for the signal of about $30 \%$. While this is higher than for the case of a light $H^{+}$(see Section 3.1), this is still quite low and an improvement should be possible with more optimized trigger algorithms. During the preselection step, at least 4 jets are required, exactly one of which should be $b$-tagged and another one $\tau$-tagged. A veto is placed on isolated leptons, and the reconstruction of a $W$ and top quark in a kinematic fit is required. Furthermore, a first cut on the missing $E_{T}$ is placed at $50 \mathrm{GeV}$. After this selection, all other backgrounds are negligible compared to $t \bar{t}$.
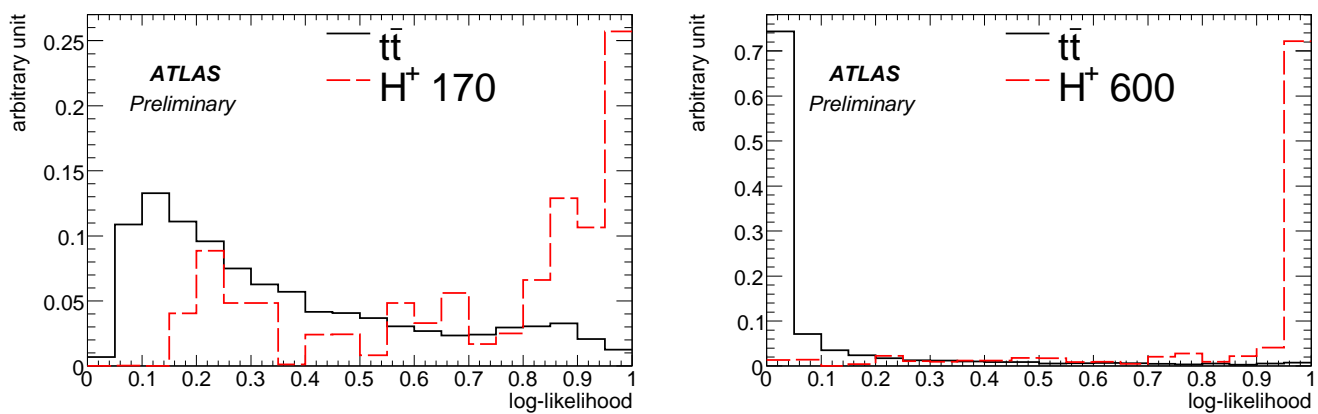

Figure 10: Likelihood value distribution for two signal mass cases and the corresponding $t \bar{t}$ background, both normalized to unity.

In the next step, a likelihood is constructed from transformations of five input variables: 1.) the $p_{T}$ of the $\tau$ jet, 2.) the missing $E_{T}, 3$.) the angle in the transverse plane between the $\tau$ jet and the missing $E_{T}$, 4.) the $p_{T}$ ratio between the $\tau$ jet and the hardest jet in the event not reconstructed as originating from the top quark, and 5.) the sum of $p_{T}$ of all jets in the event (except for the $\tau$ jet). The resulting likelihood distribution for two mass cases is shown in Figure 10. As expected, the separation between signal and background is large for very heavy $\mathrm{H}^{+}$. For an event to be kept, the likelihood value is required to be higher than $0.9\left(0.95\right.$ if $\left.m_{H^{+}}>350 \mathrm{GeV}\right)$.

For the remaining events, the mass of the $H^{+}$candidate is reconstructed, as shown in Figure 11 for three mass cases. These distributions are then used to extract the significance of the signal 

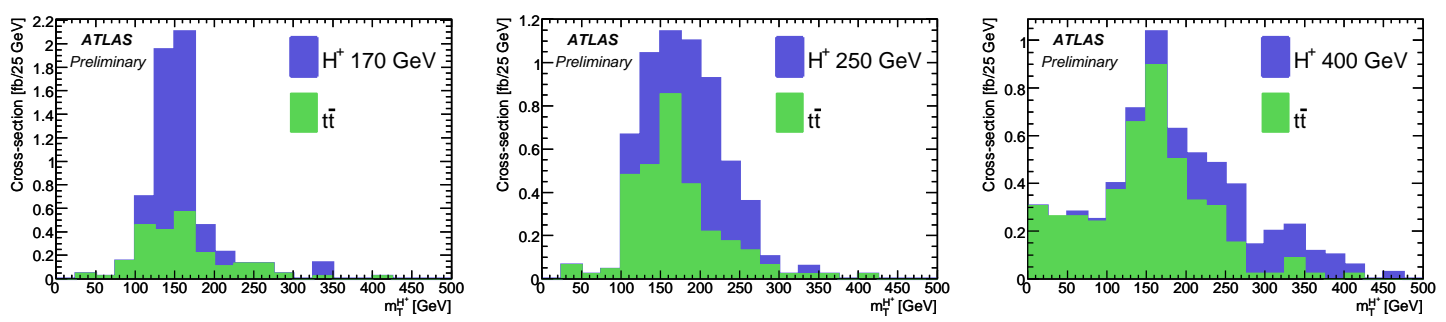

Figure 11: The reconstructed transverse mass of the $H^{+}$candidate for signal and background normalized to their cross sectios for $\tan \beta=35$, and stacked on top of each other.

using the profile likelihood method. The resulting discovery and exclusion potential is shown in Figure 12 . The discovery sensitivity covers a region in $m_{H^{+}}$up to around $350 \mathrm{GeV}$ and high $\tan \beta$. However, an $\mathrm{H}^{+}$could be excluded in a sizeable region of the MSSM parameter space.
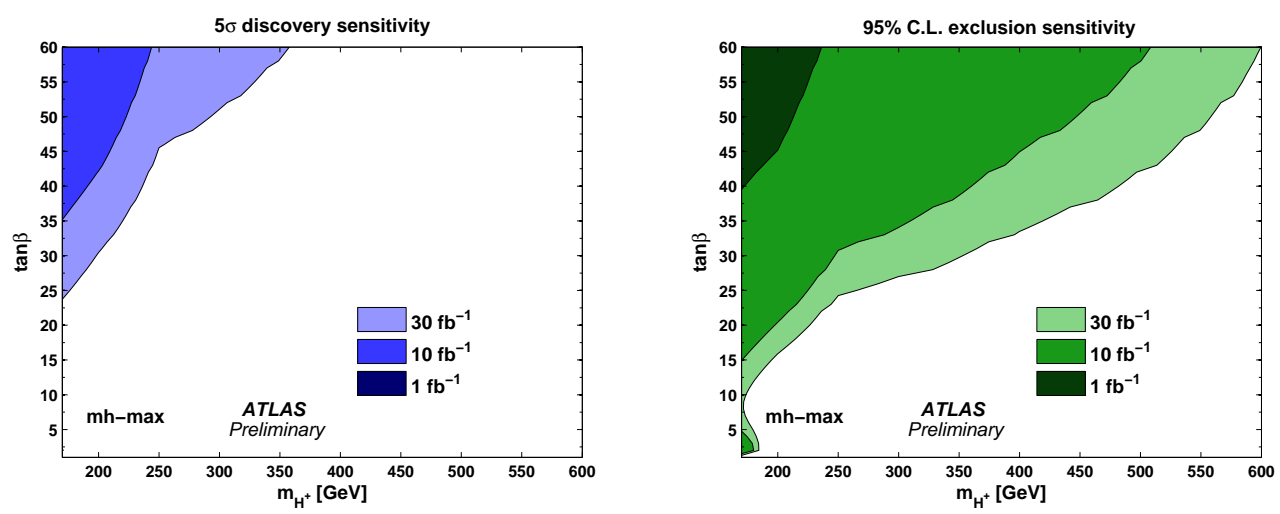

Figure 12: ATLAS $H^{+}$discovery and exclusion sensitivity for searches in the $g g / g b \rightarrow t[b] H^{+}, H^{+} \rightarrow \tau v$ mode for the $m_{h}$-max scenario. Dominant systematic and statistical uncertainties are included (see Section 5).

\section{2 $H^{+} \rightarrow t b$}

This channel has been previously studied with a parametrized detector simulation [19] and has now been reinvestigated with a realistic detector simulation. It is characterized by a high jet multiplicity which can be problematic in terms of the combinatorial background when trying to reconstruct the event. Its main strength are the 3-4 $b$ jets in the event, and a lepton which can be triggered on. Additionally, the event signature consists of two $W$ and two top quarks, which can both be reconstructed (one of each only in the transverse plane). The main backgrounds to this channel are $t \bar{t}+\mathrm{jets}, t \bar{t}+b$ and $t \bar{t}+b \bar{b}$.

Events are triggered via a ( $\tau$ jet or lepton) plus missing $E_{T}$ signature. In the preselection, an isolated lepton is required, and at least 5 jets, three of them $b$-tagged. Additionally, the successful reconstruction of the leptonic $W$ is required.

Then, a combinatorial likelihood is reconstructed from eight input variables (invariant masses of composite objects, angular relations, and the transverse momentum of the $H^{+}$candidate). The 
aim is mainly to suppress the combinatorial background, the physical background is only affected to a lesser extent. The lepton and the jets are randomly assigned to the composite objects in the event, and the combination with the highest likelihood is kept. A cut of 0.7 is placed on this likelihood value. This is followed by requiring $4 b$-tagged jets in the event.
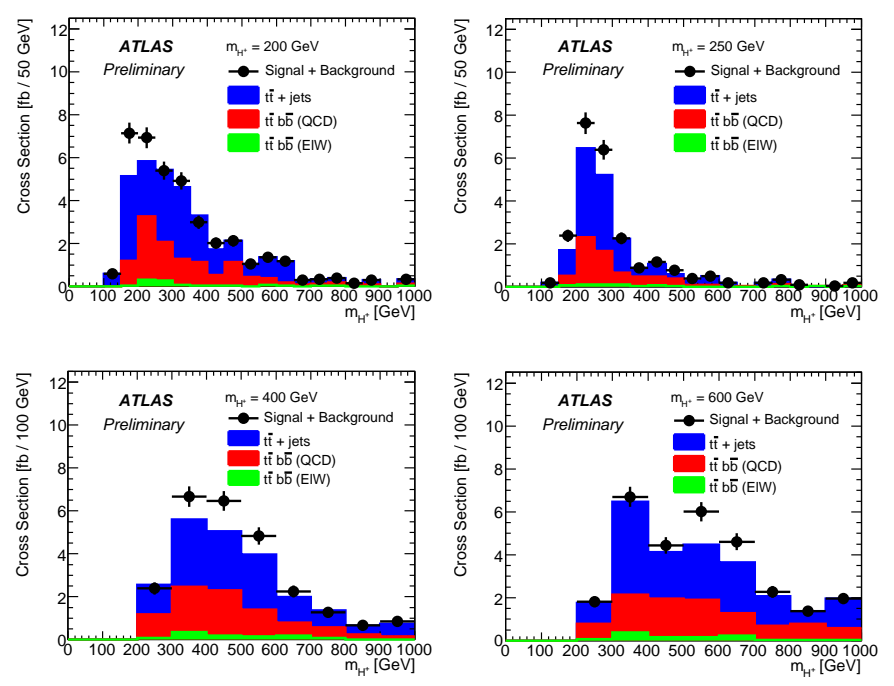

Figure 13: Mass of the reconstructed $\mathrm{H}^{+}$candidate for signal and background. For purposes of illustration, the value of $\tan \beta$ has been chosen such that the pure statistical value of the significance is 5 .

In the next step, a selection likelihood is defined aiming at the suppression of the physical background which is mostly $t \bar{t}+$ jets. However, it turns out that larger background samples than currently produced with a realistic detector simulation are needed in order to reach a performance comparable to the studies using a parametrized detector simulation. For the remaining events, the mass of the $H^{+}$candidate is shown in Figure 13. This distribution is then used to extract the significance of the signal.

The result of this study is that this channel does not offer a discovery potential in the MSSM. However, it contributes to the combined $\mathrm{H}^{+}$sensitivity and could serve for measuring the $\mathrm{H}^{+}$ mass once discovered in another channel. Additionally, it is of course sensitive for (known and unknown) models in which the cross section of the process under investigation is much higher than in the MSSM.

\section{Combined Results}

For the $t \bar{t}$ background, a total systematic uncertainty of $10 \%$ is assumed, following the background study results of $t \bar{t}$ control samples [20]. For the signal, the dominant theoretical (on the $t \bar{t}$ cross section and the branching ratio $t \rightarrow b H^{+}$, on the $g g / g b \rightarrow t b H^{+}$cross section and on the $H^{+}$ branching ratios) and experimental uncertainties (jet energy scale, tagging and identification efficiencies, energy/momentum resolution, luminosity measurement) have been taken into account, leading to uncertainties between $20-40 \%$, depending on the $H^{+}$channel. The dominant uncertainty is the jet energy scale (for quark and gluon jets as well as $\tau$ jets). 

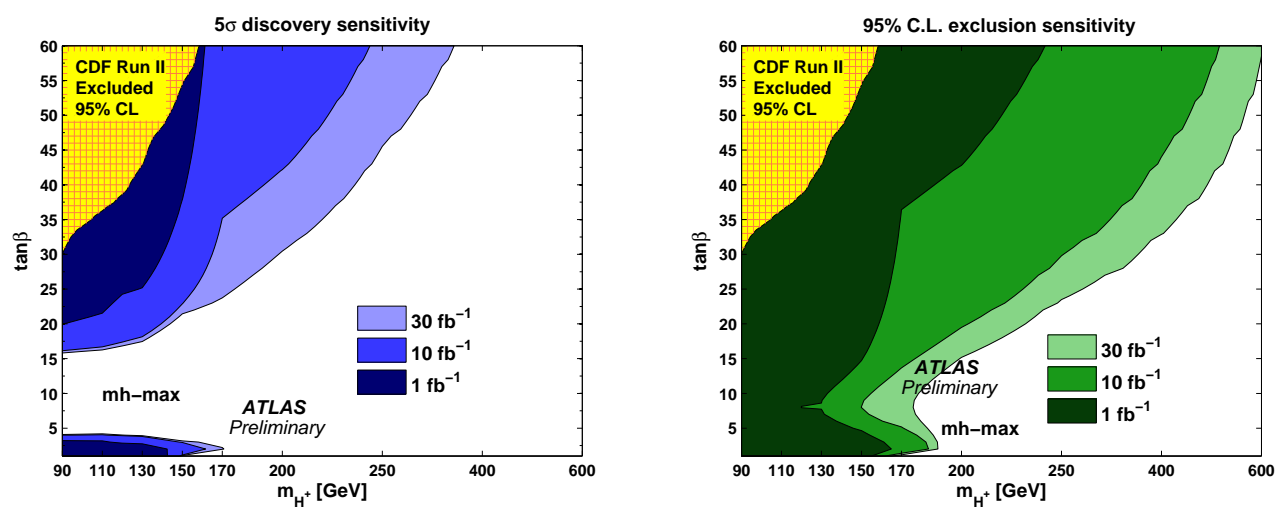

Figure 14: ATLAS combined $H^{+}$discovery and exclusion sensitivity for the $m_{h}$-max scenario.
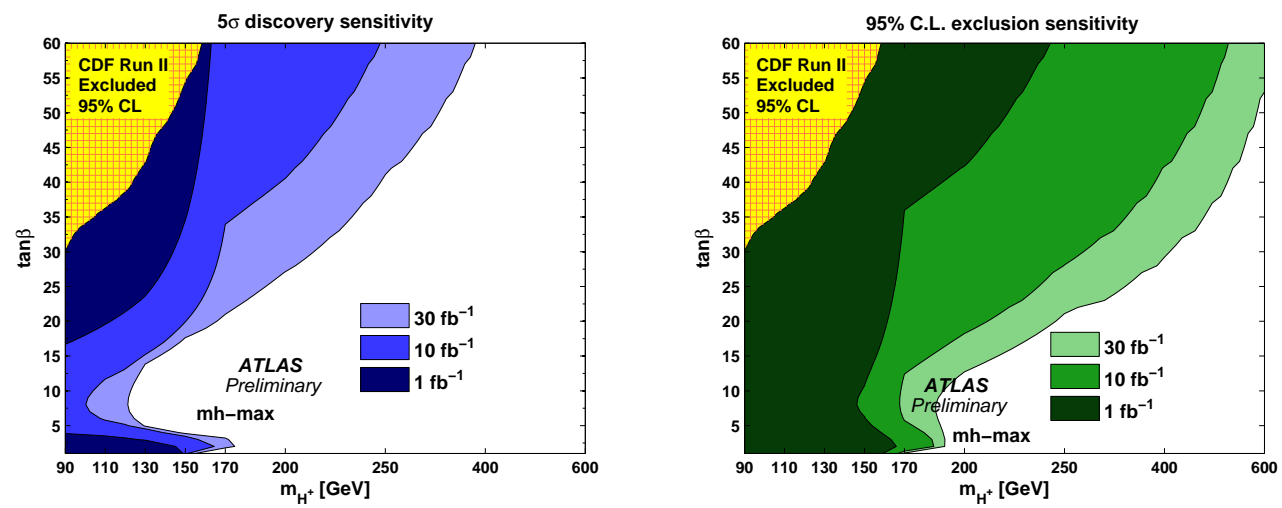

Figure 15: ATLAS combined $H^{+}$discovery and exclusion sensitivity for the $m_{h}$-max scenario neglecting uncertainties due to the finite number of simulated events.

In the following, the combined discovery sensitivity for a particular MSSM scenario, $m_{h^{-}}$ max, and for model-independent scenarios are presented. The different $\mathrm{H}^{+}$channels have been combined using the profile likelihood method [14]. Discovery contours correspond to a significance of $5 \sigma$, exclusion contours to a $95 \%$ confidence level.

\section{$5.1 m_{h}$-max}

The combined discovery contour for the $m_{h}$-max scenario is shown in Figure 14 . The sensitivity exceeds the Tevatron exclusion already with a few $\mathrm{fb}^{-1}$ of (well-understood!) data, corresponding to a few months of running at low luminosity. A sizeable region in the parameter space is covered, in particular in terms of exclusion. However, the intermediate $\tan \beta$ region (around 3-16) is not covered for discovery, and heavy $\mathrm{H}^{+}$are hard to access due to their low production cross section. This is partially due to the number of simulated events which is relatively small leading to a large statistical uncertainty when extrapolating to high integrated luminosities.

To illustrate this effect, Figure 15 shows the same contours when neglecting the uncertainties due to limited MC statistics, showing what might be possible in a study repeated on a future larger 
event production. Here, the intermediate $\tan \beta$ region is covered for light $H^{+}$and the reach also extends to higher $H^{+}$masses.

\subsection{Model-Independent}
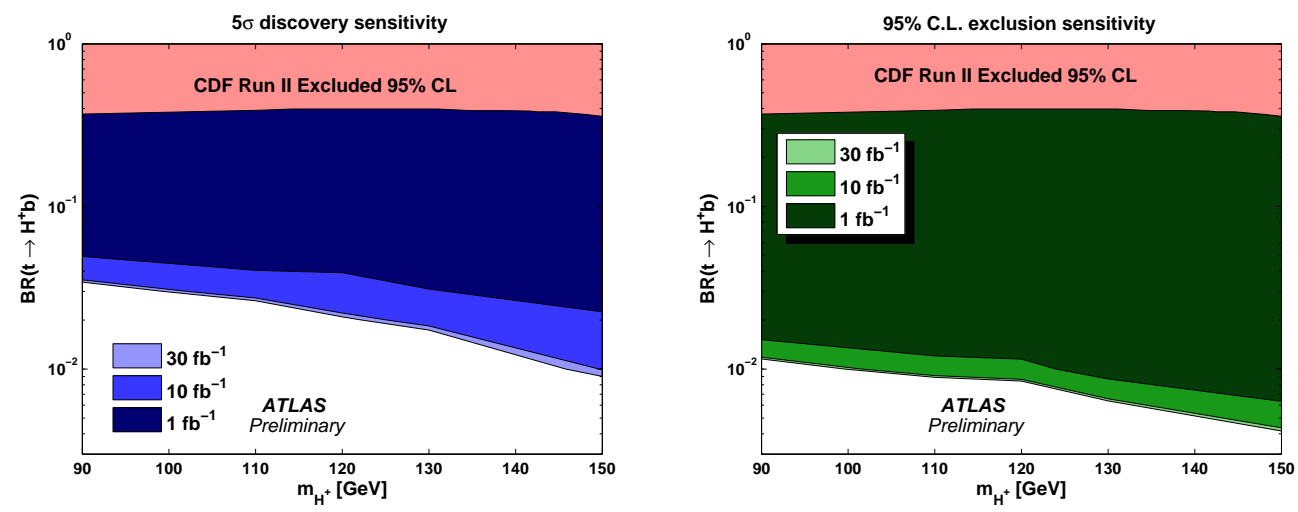

Figure 16: ATLAS combined light $H^{+}$discovery and exclusion sensitivity in terms of the branching ratio $t \rightarrow b H^{+}$.
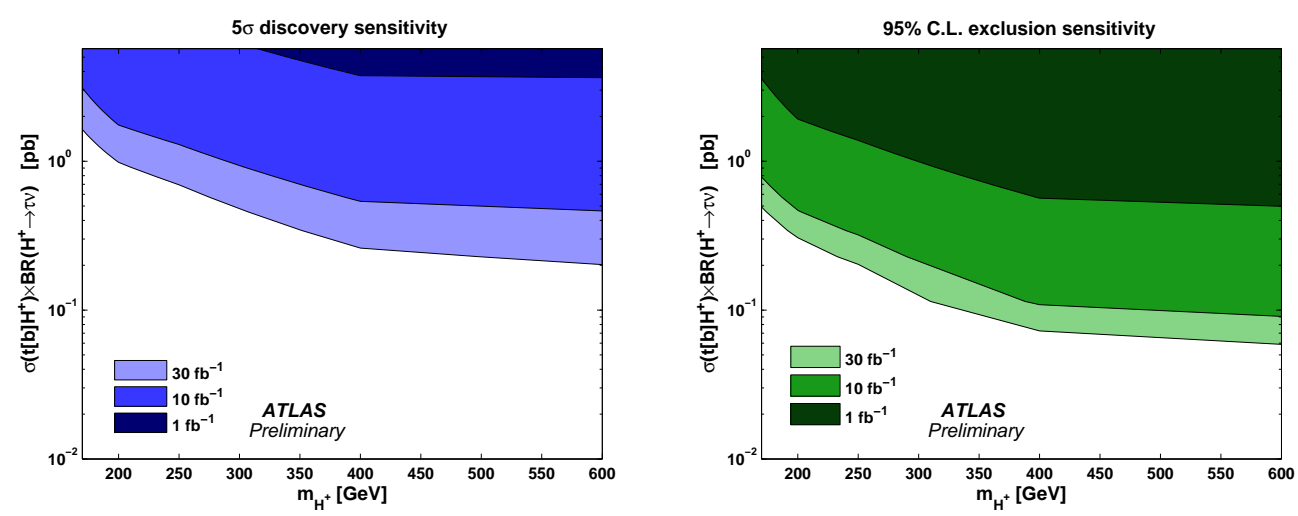

Figure 17: ATLAS combined heavy $H^{+}$discovery and exclusion sensitivity in terms of the cross section $\sigma\left(t b H^{+}\right) x B R\left(H^{+} \rightarrow \tau \nu\right)$.

Figure 16 shows the discovery and exclusion contour in terms of the branching ratio $t \rightarrow H^{+} b$ as a function of $m_{H^{+}}$. With one year of low luminosity data, it will be possible to discover the charged Higgs boson if $\operatorname{BR}\left(t \rightarrow H^{+} b\right)$ is larger than about 1-3\%, and to exclude it even if this branching ratio is well below the percent level. Similar contours for a heavy $H^{+}$are presented in Figure 17; here the y-axis shows the cross section for the process $g g / g b \rightarrow t[b] H^{+} \rightarrow t[b] \tau v$. Sensitivity is given for a cross section of the order of $0.1 \mathrm{pb}$. Both figures are model-independent in the sense that they can be interpreted in the context of any MSSM, other SUSY, or even non-SUSY scenario.

\section{Conclusions and Outlook}

The ATLAS potential for discovering or excluding the existence of a charged Higgs boson in two 
different MSSM scenarios has been reported for five different final states of the $H^{+}$signal. It should be noted that these are only the most recently updated studies. Additionally, further ATLAS $\mathrm{H}^{+}$ sensitivity studies in other channels as well as investigations to address the measurement of physics properties of the $\mathrm{H}^{+}$have been performed. Significant improvements of present day constraints can already be achieved with limited data (less than $1 \mathrm{fb}^{-1}$, about one month at low luminosity at the LHC) although it may not qualify as early physics due to its dependency on higher level reconstruction objects.

The combined performance yields a discovery reach for $10 \mathrm{fb}^{-1}$ which covers $\tan \beta$ values down to 20 and up to 4 for all charged Higgs boson masses up to about $150 \mathrm{GeV}$ in the $m_{h}$-max scenario. In the intermediate $\tan \beta$ region (around $\tan \beta=7$ ), no discovery sensitivity is present, but a charged Higgs boson could be excluded in this region. It is likely that a future study based on a large amount of simulated events will improve the situation, in particular by giving access to the intermediate $\tan \beta$ region.

For a heavy $H^{+}$, the sensitivity reaches from $\left(m_{H^{+}}=200 \mathrm{GeV}, \tan \beta=28\right)$ to $\left(m_{H^{+}}=350 \mathrm{GeV}\right.$, $\tan \beta=58$ ) for an integrated luminosity of $30 \mathrm{fb}^{-1}$. Additionally, the model-independent discovery reach for a charged Higgs boson as a function of its production cross section has been evaluated. A light $H^{+}$sensitivity for a $\operatorname{BR}\left(t \rightarrow H^{+} b\right)$ down to the percent level is given for discovery, and well below that level for exclusion. For a heavy $H^{+}$decaying to $\tau \nu$, sensitivity is given for cross sections of the order of $0.1 \mathrm{pb}$.

The results presented give confidence that the LHC and the ATLAS detector will be able to probe an extended Higgs sector over a sizable region of the MSSM parameter space. For a high SUSY mass scale, the charged Higgs boson could be the first signal of the existence of New Physics.

\section{Acknowledgements}

I would like to thank my colleagues from the ATLAS $H^{+}$subgroup, the Higgs group and their convenors for their direct contribution to the material presented in this talk, as well as for their support in preparing this note and the corresponding talk. I would also like to express my gratitude towards the whole ATLAS Collaboration as everyone has at least indirectly contributed to the presented results.

\section{References}

[1] ALEPH, DELPHI, L3, OPAL, and the LEP Higgs Working Group, hep-ex/0107031 (2001)

[2] A. Abulencia et al., CDF Collaboration, Phys. Rev. Lett. 96 (2006) 042003

[3] F. Winklmeier, ATLAS Trigger Performance and Initial Running, this volume

[4] C. Potter, Trigger Strategies, this volume

[5] R. Brenner, Status and Performance of the ATLAS Tau Trigger, this volume

[6] A. Saavedra, Tau Lepton Reconstruction and Identification with the ATLAS Detector, this volume

[7] N. G. Piacquadio, b-tagging at LHC, this volume

[8] ATLAS Collaboration, Expected Performance of the ATLAS Experiment, Detector, Trigger and Physics, CERN-OPEN-2008-020 (2008) 
[9] A. Sopczak, Cross-sections and Branching Ratios for H+ Searches, this volume

[10] S. Heinemeyer et al., FeynHiggs version 2.6.2, hep-ph/9812320, hep-ph/9812472, hep-ph/0212020, hep-ph/0611326

[11] R. Bonciani et al., Nucl Phys B 529 (1998) 424-450

[12] C. Biscarat, M. Dosil, ATL-PHYS-2003-038 (2003)

[13] E. Coniavitis, Light $\mathrm{H}+$ searches in the Hadronic Mode with ATLAS, this volume

[14] O. Vitells, Statistical Combination of charged Higgs channels in ATLAS, this volume

[15] O. Vitells, Charged Higgs searches in the leptonic tau channel, this volume

[16] T. Ehrich, Searches for Light H+ Decaying to a Hadronic Tau in the One Lepton Mode with ATLAS, this volume

[17] E. Boos, T. Plehn, Phys. Rev. D 69 (2004) 094005

[18] B. Mohn, M. Flechl, J. Alwall, ATL-PHYS-PUB-2007-006 (2007)

[19] K. Assamagan, N. Gollub, SN-ATLAS-2004-042 (2004)

[20] T. Vickey, Data-driven Methods for the Estimation of ttbar Backgrounds to Charged Higgs Searches, this volume 